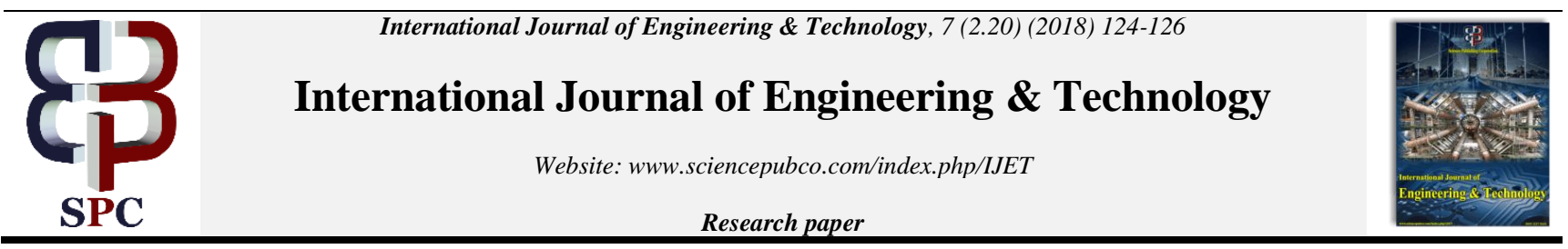

\title{
A Versatile and Cost Effective Multimodal Wheelchair
}

\author{
A.Sampath Dakshina Murthy ${ }^{*}$, Dr.Rudra Pratap Das², Dr.S.Koteswara Rao ${ }^{3}$ \\ ${ }^{1,2}$ Department of Electronics and Communication Engineering, Vignan's Institute of Information \\ Technology, Visakhapatnam, A.P, India \\ ${ }^{3}$ Department of Electronics and Communication Engineering, K.L.University, Guntur, A.P, India \\ *Email: sampathdakshinamurthy@gmail.com
}

\begin{abstract}
The aim of this endeavor is to design a wheelchair to suit to the needs of such persons .By incorporating temperature and humidity sensor, IR sensor, FSR sensor and voice recognition sensor/module the device is quite versatile in attending to the basic problems encountered .After testing ,it was found that this apparatus helps in avoiding obstacles. If allows the person to use his tongue of required. The additional benefit is to have voice recognition for movement .Cost effectiveness is yet another merit of the proposed system.
\end{abstract}

Keywords: FSR sensor; Arduino; Voice recognition sensor; IR sensor; Wheel chair.

\section{Introduction}

An intelligent wheel chair comprising of sensors such as temperature and humidity [1]. IR sensors [2], FSR sensor [3], voice recognition sensor [4] and microcontroller [5] along with arduino board [5]. It can be used by physically challenged or elder person to move inside the home effort less without any help of others. Offen the elders forget the location of different rooms and physically challenge an counter difficulty moving the wheel chair themselves .By using temperature and humidity sensor working can be given to avoid extreme temperature points as in the kitchen .The IR sensor helps in locating obstacles stopping the wheel chair .FRS helps the paralyzed people who can move only tongue; Different pressures stripes placed in the mouth help in movement of chair depending on the applied pressure .The voice recognition module facilities movement into different rooms ,by moving the person pronounce a suitable word predefine in the system. All these sensing modules are currently controlled by a switching mechanism so that the user can have an automatic chose [6] .This wheel chair is not only cost effective but less wait, high durability power saving advantage [7].

\section{Conditions of Movement}

The proposed wheel chair has five conditions modes of operations
1. Moving forward to the front of user
2. Moving backward to the rear
3. Moving turning to the right
4. Moving turning to the left
5. Static halt condition

\section{Principle of Operation}

The temperature and humidity module provide an input to AT89S5S microcontroller circuit kit. So that different temperature is detected and warning is mode against high temperatures. Any obstacle on the path is defected by the IR sensor which stops the wheel chair[8] and alerts the user. The FSR pressure strips wait for the application of pressure by the user in figure 3.The movements such as forwarded and back warded different amount pressure figure 1 . The basic system starts after application of supply voltage and remain in stand by condition shown by a led. Voice recognition sensor can have the facility choosing the speed in two levels slow are fast figure 2 .For instance the user need to move in a short distance are object nearby slow speed may be selected shown figure 4 . All these modules are interface placed on board along with arduino and microcontroller shown figure 5 .

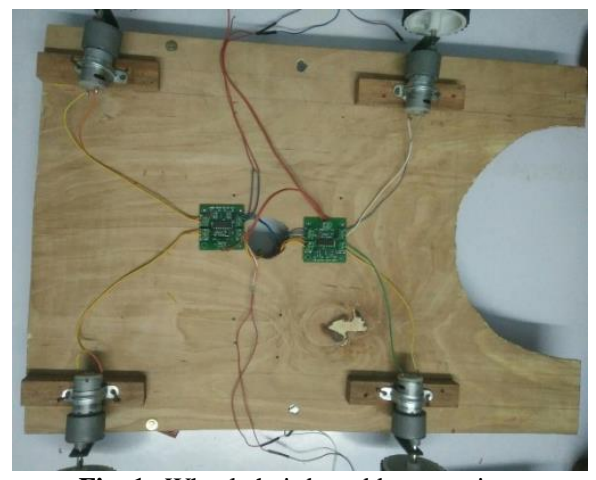

Fig. 1: Wheel chair board bottom view

Copyright $\odot 2018$ Authors. This is an open access article distributed under the Creative Commons Attribution License, which permits unrestricted use, distribution, and reproduction in any medium, provided the original work is properly cited. 

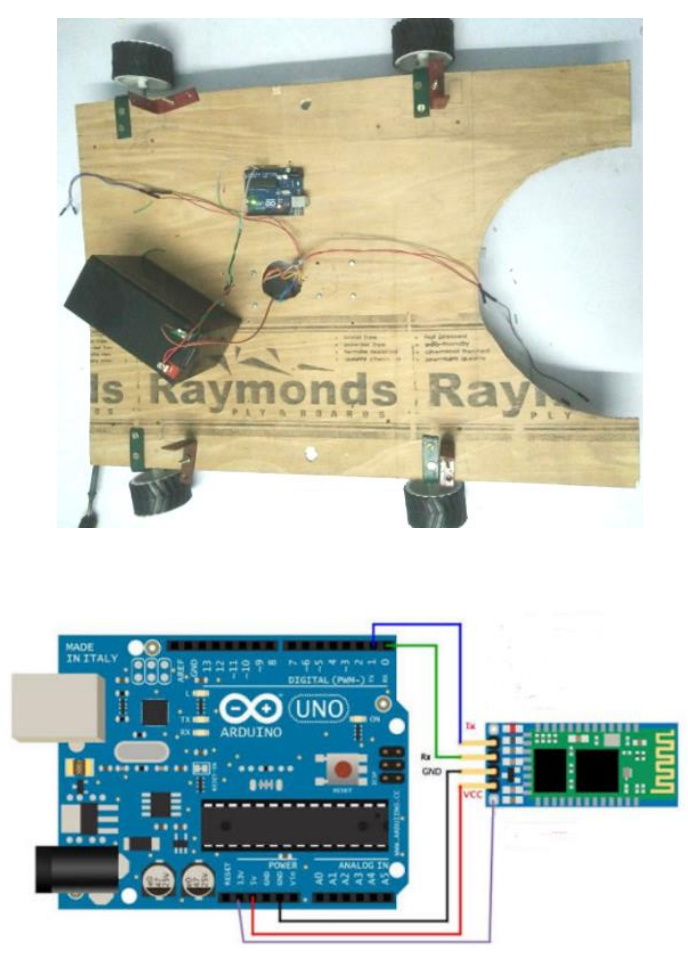

Fig. 2: Wheel chair board top view connected with voice module


Fig. 3: Wheel chair board top view connected with FSR module

\section{Interfacing of sensors}

The output pin of IR sensor is connected to pin 1.1 of microcontroller. The output of infrared sensor module is indicated by led .DTH 11 sensors are connected by three wires $\mathrm{V}_{c c}$, data, GND.variable dth_rh and dth_temp are the important variables. FSR is made in two layers separated by a pressure. These are basically resistors and they are two pins one is connected to $5 \mathrm{~V}$ other to $A_{0}$ which is observed in figure 3 .Voice Bluetooth module $\mathrm{T}_{\mathrm{x}}, \mathrm{R}_{\mathrm{X}}, \mathrm{V}_{\mathrm{cc}}$ and GND shown in figure 2. LM293 service of motor drivers can be used as interfacing with arduino and microcontroller .Motor IC L293D is selected having 16 pins it works on con- cept of Hbridge which allow the voltage which shown in either direction .In single chip they are two Hbridge circuit which can rote using two DC motors.

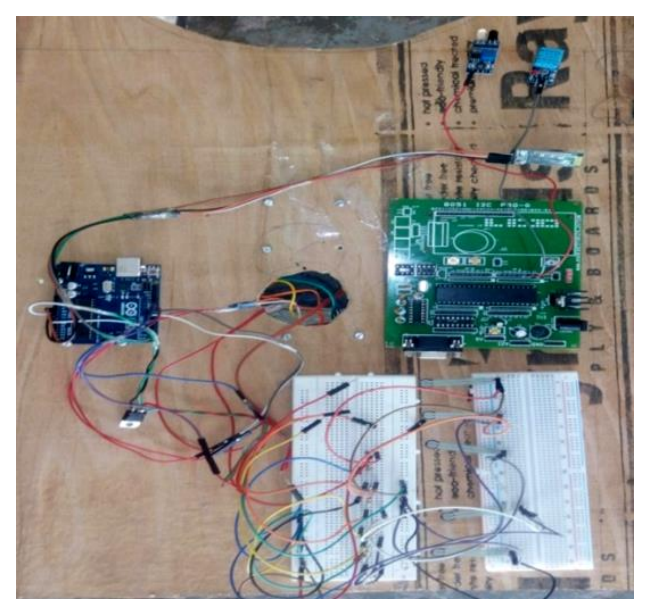

Fig. 4: Wheel chair board top view connected with all components

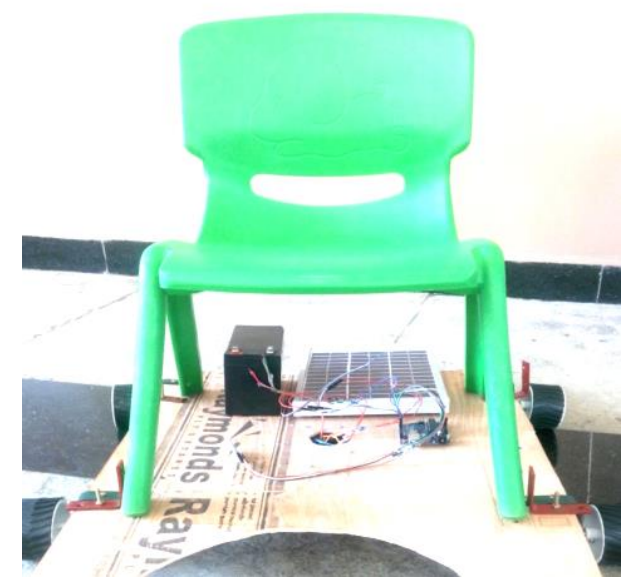

Fig. 5: Wheel chair board top view connected with all components Multipurpose multi module wheel chair

\section{Conclusion}

Due to the multipurpose multimodal operation these wheels chair as many unique advantages. Design is aimed at reduced of the cost of the manufacture compared with those available in the market future addition of sensors and solar power can make this wheel chair even more versatile.

\section{References}

[1] Simpson, Richard C. "Smart wheelchairs: A literature review." Journal of rehabilitation research and development 42, no. 4 (2005): 423.

[2] HAN, Jian, Cai-hua MA, Deng-hui PANG, and Jin-zhao WAN. "Design of intelligent multifunctional bed based on Android." Electronic Design Engineering 3 (2017): 033.

[3] Dowling, Ariel V., Valerie Eberly, Somboon Maneekobkunwong, Sara J. Mulroy, Philip S. Requejo, and Joseph T. Gwin. "Telehealth monitor to measure physical activity and pressure relief maneuver performance in wheelchair users." Assistive Technology 29, no. 4 (2017): 202-209.

[4] Viswanathan, Pooja, Richard C. Simpson, Geneviève Foley, Andrew Sutcliffe, and Julianne Bell. "Smart wheelchairs for assessment and mobility." In Robotic Assistive Technologies, pp. 161194. CRC Press, 2017.

[5] Shruti, Km, Shelej Khera, Manoj Kumar, and Vikrant Verma. "A Microcontroller Based Multimodal Wheelchair Prototype For The 
Disabled." Journal of Instrumentation and Innovation Sciences 2, no. 1, 2 (2017).

[6] Raiyan, Zannatul, Md Sakib Nawaz, AKM Asif Adnan, and Mohammad Hasan Imam. "Design of an arduino based voicecontrolled automated wheelchair." In Humanitarian Technology Conference (R10-HTC), 2017 IEEE Region 10, pp. 267-270. IEEE, 2017.

[7] Elon, Sarah Ruth, Kip Darrin Alder, and Stephen J. Antonishak. "Wheelchair housing." U.S. Patent Application 29/556,157, filed May 23, 2017.

[8] Elon, Sarah Ruth, Kip Darrin Alder, and Michael A. Grant. "Wheelchair compartment." U.S. Patent Application 29/556,158, filed October 31, 2017. 